\section{AB1299 AN ONGOING ANTICENTROMERE ANTIBODY RESPONSE ASSOCIATES WITH PROGRESSION TOWARDS SYSTEMIC SCLEROSIS}

Nina van Leeuwen ${ }^{1}$, Maaike Boonstra ${ }^{1}$, Jaap Bakker ${ }^{2}$, Corrie Wortel ${ }^{1}$, Hans Ulrich Scherer ${ }^{1}$, Rene Toes ${ }^{1}$, Thomas Huizinga ${ }^{1}$, Jeska de Vries-Bouwstra ${ }^{1}$.

${ }^{1}$ Leiden University Medical Center, Rheumatology, Leiden, Netherlands; ${ }^{2}$ Leiden, Clinical chemistry, Leiden, Netherlands

Background: Although some dated studies suggest a possible association between clinical characteristics and isotypes of anticentromere antibodies (ACA) in patients with systemic sclerosis (SSc), characteristics of ACA have not been described thoroughly in SSc.

Objectives:

1. Describe characteristics of anticentromere antibody (ACA) response in a large cohort of SSc patients.

2. Evaluate differences between ACA response between SSc patients fulfilling ACR 2013 criteria and those with very early diagnosis of SSc (VEDOSS).

Methods: ACA IgG, IgM and IgA levels were assessed in serum samples of patients visiting the Leiden SSc care pathway and who were IgG ACA+ at baseline. Patients had to fulfil either the ACR criteria or the VEDOSS criteria. Differences in isotype expression and levels between the two groups were evaluated, also with stratification for disease duration. Furthermore, in the SSc group we determined correlations between isotypes and disease duration and evaluated if isotype positivity was associated with clinical manifestations.

Results: ACA characteristics were measured in 167 ACA lgG+ patients. Thirty-five patients $(21 \%)$ fulfilled the VEDOSS criteria and $132(79 \%)$ the ACR criteria. ACA IgG+ patients displayed a broad isotype usage with $75 \%$ being ACA IgA+ and $68 \%$ being ACA lgM+ in serum. Patients within the SSc group showed higher ACA IgG levels and a higher percentage of ACA IgM positivity compared to the VEDOSS patients. Notably, these findings remained valid after stratification for disease duration, demonstrating that VEDOSS patients that did not progress to SSc within at least 5 years had lower ACA IgG levels and were less frequently positive for ACA IgM. In the SSc group, moderate correlations between isotypes were found; ACA IgM positivity was associated with occurrence of digital ulcers $(p=0.02)$.

\begin{tabular}{|c|c|c|c|}
\hline & $\begin{array}{c}\text { Total group } \\
(n=167)\end{array}$ & $\begin{array}{c}\begin{array}{c}\text { Very early SSC } \\
(n=35)\end{array} \\
\end{array}$ & $\begin{array}{c}\mathrm{SSc} \\
(\mathrm{n}=132)\end{array}$ \\
\hline Female, $n(\%)$ & $150(90)$ & $34(92)$ & $116(89)$ \\
\hline Age, mean (SD) & $58(13)$ & $55(15)$ & $58(13)$ \\
\hline Since RP, median(IQR) & $12(5-25)$ & $10(4-26)$ & $13(5-25)$ \\
\hline Since non RP, median(IQR) & $4(1-11)$ & $2(1-5)$ & $5(1-12)$ \\
\hline mRSS, median (IQR) & $2(0-4)$ & $0(0-0)$ & $3(0-5)$ \\
\hline Teleangiectasia, $n(\%)$ & $117(70)$ & $7(20)$ & $110(83)$ \\
\hline Digital ulcers, $\mathrm{n}(\%)$ & $33(20)$ & $0(0)$ & $33(25)$ \\
\hline ILD on HRCT, $n(\%)$ & $13(8)$ & $0(0)$ & $13(10)$ \\
\hline $\mathrm{PAH}, \mathrm{n}(\%)$ & $12(7)$ & $0(0)$ & $12(9)$ \\
\hline \multicolumn{4}{|l|}{ ACA characteristics } \\
\hline IgA positivity, n(\%) & $125(75)$ & $26(72)$ & $99(76)$ \\
\hline IgA level [aU/mL], median (IQR) & $73(39-139)$ & $76(35-130)$ & $73(37-142)$ \\
\hline IgM positivity, n(\%) & $114(68)$ & $18(50)$ & $96(73)^{*}$ \\
\hline IgM level [aU/mL], median (IQR) & $64(1-527)$ & $21(0-487)$ & $65(4-527)$ \\
\hline IgG level [aU/mL], median (IQR) & $361(156-919)$ & $197(94-530)$ & $478(183-1010)^{* *}$ \\
\hline
\end{tabular}

Figure 1

Conclusion: We observe a clear difference in the quality of the centromere-specific immune response between VEDOSS patients and SSc patients, also when stratifying for disease duration. The higher ACA IgG levels and presence of ACA IgM in SSc patients indicates that the ACA response in SSc is more pronounced showing signs of ongoing activity. These data indicate that the ACA B cell response is potentially relevant in disease development. In addition, ACA isotype expression and ACA IgG levels might contribute to better identify VEDOSS patients at risk for SSc development.

Disclosure of Interests: Nina van Leeuwen: None declared, Maaike Boonstra: None declared, Jaap Bakker: None declared, Corrie Wortel: None declared, Hans Ulrich Scherer Grant/research support from: Sanofi, BMS, Rene Toes Grant/research support from: Sanofi, Thomas Huizinga Consultant for: Merck, UCB, Bristol Myers Squibb, Biotest AG, Pfizer, GSK, Novartis, Roche, Sanofi-Aventis, Abbott, Crescendo Bioscience Inc.,
Nycomed, Boeringher, Takeda, Zydus, Epirus, Eli Lilly, Jeska de VriesBouwstra: None declared

DOI: 10.1136/annrheumdis-2019-eular.3029

\section{AB1300 AUTOIMMUNE/INFLAMMATORY SYNDROME INDUCED BY ADJUVANTS DUE TO SILICONE BREAST IMPLANT AND RHEUMATIC DISEASES:}

Olga Lidia Vera Lastra ${ }^{1}$, Maria del Pilar Cruz Dominguez ${ }^{1}$, Luis Javier Jara ${ }^{2}$, Gabriel Medrano Ramirez ${ }^{3}{ }^{1}$ Unidad Medica de Alta Especialidad Hospital de Especialidades Dr Antonio Fraga Mouret Centro Medico Nacional La Raza, Internal Medicine, Mexico City, Mexico; ${ }^{2}$ Unidad Medica de Alta Especialidad Hospital de Especialidades Dr Antonio Fraga Mouret Centro Medico Nacional La Raza, Direccion de investigacion y educacion, Mexico City, Mexico; ${ }^{3} \mathrm{Hospital}$ General de Mexico, Reumathology, Mexico City, Mexico

Background: Silicone is considered biologically inert; thus it has been employed in many medical devices and nowadays is commonly used in plastic surgery for mammary prosthesis. The most common adverse reaction after silicone breast implant $(\mathrm{SBI})$ is capsular contracture due to a foreign body reaction. Autoinmune Rheumatic Diseases (ARD) have been associated with $\mathrm{SBI}$ or siliconosis as part of the spectrum of Autoimmune/Inflammatory Syndrome Induced by Adjuvants (ASIA). Silicone is an adjuvant that may 'bleed' and subsequently may be a chronic stimulus to the immune.

Objectives: To determine the frequency of rheumatic diseases (ARD) and associated factors in patients with ASIA induced by SBI (ASIA-SBI).

Methods: This study was performed between 2012 to 2018 in a tertiary hospital from a cohort of 200 patients with ASIA (associated of oily substance, vaccines and siliconosis according to Shoenfeld criteria). We selected those patients with ASIA induced by $\mathrm{SBI}$ and clinical manifestations of any autoimmune disease. The clinical manifestations of ARD were evaluated and classified according to The American College of Rheumatology or EULAR criteria for some rheumatic diseases. We also investigated family history of autoimmune diseases, tobacco smoking coexisting allergies and comorbidities such as hypothyroidism, depression, anxiety and others. Main autoantibodies, histological findings were also evaluated. We used descriptive statistics for analysis.

Results: There were 30 women patients with mean age 48.5 \pm 16.346 years old, mean disease duration of disease $6 \pm 5.5$ years. The mean time of appearance of clinical manifestations after SBI was $8 \pm 4.9$ years. The rheumatic diseases (RD) or ARD were Systemic sclerosis (SSc) 7, Fibromyalgia (FM) 6, Systemic lupus erythematosus (SLE) 3, rheumatoid arthritis (RA) 6, overlap syndrome 2 (SSc plus SS and SLE plus SSc), Sjogren syndrome (SS) 1, Takayasu arteritis 1, Still disease 1, tunnel carpal syndrome 1, antiphospholipid syndrome 1 and angioedema and urticaria 1. We found family history of rheumatic disease in $40 \%$, allergic history ( food, drugs) in $40 \%$ and smoking in 50\%. Hypothyroidism was observed in $30 \%$ and anxiety $30 \%$. Patients were treated according to the rheumatic disease. Ten cases had their prostheses removed because extracapsular ruptured implants, with re-implantation of new prosthesis in 8 patients. Two patients experienced improvement of complaints after explantation of the implant.

Conclusion: We found a prevalence of ASIA associated to SBI of $15 \%$. The main RD were SSc, FM and RA. We observed an association with family history, allergies and smoking in these patients.

Further studies are necessary to determine if SBI has a cause -effect relationship in the rheumatic disease manifestations.

\section{REFERENCES}

[1] Jara LJ, García-Collinot G, Medina G, Cruz-Dominguez MD, Vera-Lastra O, Carranza-Muleiro RA, Saavedra MA. Severe manifestations of autoimmune syndrome induced by adjuvants (Shoenfeld's syndrome). Immunol Res. 2016 Jul 13

[2] Colaris MJ, de Boer M,van der Hulst RR, Cohen Tervaert JW,Two hundreds cases of ASIA syndrome following silicone implants: a comparative study of 30 years and a review of current literature. Immunol Res. 2016 Jul 13.

[3] Watad A, Quaresma M, Brown S, Cohen Tervaert JW, Rodríguez-Pint I, Cervera R, Perricone C, Shoenfeld Y. Autoimmune/inflammatory syndrome induced by adjuvants (Shoenfeld's syndrome) - An update. Lupus. 2017 Jan 1:961203316686406.

Disclosure of Interests: None declared DOI: 10.1136/annrheumdis-2019-eular.7590 